\title{
Prevalence of celiac disease: a multicenter trial in pediatric population from five urban districts of Argentina
}

\author{
Mabel Mora, $M D^{a}$, Néstor Litwin, $M D^{b}$, María del Carmen Toca, $M D^{c}$, \\ María Inés Azcona, $M D^{a}$, Rossana Solís Neffa, $M D^{a}$, Fernando Battiston, $M D^{a}$, \\ María Solaegui, $M D^{a}$, Gonzalo Ortiz, $M D^{c}$, Marta Wagener, $M D^{d}$, \\ Jorgelina Olivera, $M D^{d}$, Silvia Marchisone, $M D^{e}$, Gabriela Oropeza, $M D^{e}$, \\ Cinthia Bastianelli, $M D^{f}$, Alejandra González, $M D^{g}$ and Gabriela Rezzónico, $M D^{h}$
}

\section{SUMMARY}

Introduction. No published material on the prevalence of celiac disease (CD) in the pediatric population of Argentina has been found up to date.

Objective. To estimate the prevalence of $\mathrm{CD}$ in a pediatric population (hospital-based sample) from 5 urban districts of Argentina.

Methods. In a cross-sectional descriptive study, we analyzed serum samples from 2219 children, aged 3-16 years old, which had been requested for pre-surgical tests and for physical aptitude certificates for sports in the province of Buenos Aires, and cities of Buenos Aires, Córdoba, Santa Fe and Salta. Children with a previous and accurate diagnosis of $\mathrm{CD}$ were also included. IgA class tissue transglutaminase antibodies were determined using serum samples, and those samples which turned out positive were also tested for IgA class endomysium antibodies. A small intestine biopsy was proposed for those who had a positive serology.

Results. Between May 2008 and August 2009, 29 positive serologies were found. A total of 22 duodenum biopsies were performed, and 21 turned out compatible with CD. Out of 2219 children, 7 had a previous diagnosis. A prevalence of $1.26 \%$ (1:79 children), with female gender predominance $(\mathrm{p}<0.023)$ was found.

Ninety percent of the celiac children were over 6 years old $(p<0.021)$. Silent celiac disease predominated but there was a $33 \%$ of symptomatic cases.

Conclusions. The results of the trial show a higher prevalence of $C D$ than expected. The finding of symptomatic patients (33\%) suggests the undertaking of different activities to spread the knowledge on this disease and promote the indication for serology test, to avoid complications by means of an early diagnosis.

Key words: celiac disease, prevalence, children, tissue transglutaminase, Argentina.

http:/ / dx.doi.org/10.5546/aap.2012.490

\section{INTRODUCTION}

Celiac disease is a permanent condition of gluten intolerance, characterized by damage to the mucous of the small intestine, produced as a con- sequence of an inflammatory response to the intake of gluten in genetically susceptible individuals.

Its clinical spectrum is varied and includes three forms: classical, with malabsorption syndrome, silent and atypical. Extraintestinal -often oligosymptomatic- manifestations can also be observed in atypical celiac disease, such as short stature, osteoporosis, anemia, abortions, infertility, epilepsy, ataxia, occipital cerebral calcifications, recurrent aphthous ulcers or dermatitis herpetiformis. ${ }^{1-3}$

If any of these occur, the early diagnosis of celiac disease should be considered to avoid complications. ${ }^{4-6}$ There are conditions associated to celiac disease, such as Down, Williams, Turner syndromes, and autoimmune disorders, such as type 1 diabetes, Hashimoto's thyroiditis or autoimmune hepatitis, among others. ${ }^{7,8}$

The so-called silent celiac patients present no clinic manifestation and, while they are most common in the so-called risk groups, they can also be found among the general population. Risk groups are composed by those who have family members with celiac disease or associated conditions.

The clinical spectrum of this disease has been compared to an iceberg, in which the symptomatic form is the visible part, while the atypical and silent forms, almost seven times more frequent, constitute the submerged part. ${ }^{9}$

In the 1980s, serological determinations, such as antigliadin, started being performed, spreading the knowledge on this clinical spectrum. Subsequently, other antibodies of 
greater specificity and sensitivity appeared, such as anti-endomysium (EMA) and tissue transglutaminase (tTG). EMA showed a $96 \%$ sensitivity and a specificity close to $100 \%$, while tTG has a $95-99 \%$ specificity and a $96 \%$ sensitivity in children. The technique for the latter is simpler, and therefore it is used as the method of choice in population studies. ${ }^{10}$

The diagnosis of celiac disease is confirmed through a small intestine biopsy. The symptoms, positive antibodies and intestinal damage are reversible with a gluten-free diet.

Assessing the magnitude of celiac disease requires knowledge of all its clinical manifestations, especially those hidden, for which a prevalence study in general population is the most important source of information.

In the literature there are three modalities of prevalence studies, given the clinical characteristics of celiac disease:

1. Diagnosed cases of celiac disease on population density in a determined area. These represent the first epidemiological studies, prior to the use of serology; cases were generally symptomatic, classical and their prevalence was lower. For instance, in 1950, in England and Wales, the prevalence of celiac disease was estimated at 1 every 8000 individuals. ${ }^{11}$ Subsequent studies were conducted in Italy as from 1990, with the first use of serology.

2. Studies of celiac disease cases in risk populations (family members and patients with associated conditions), and their prevalence was higher. Fasano et al., found a prevalence of 1:22 among family members of patients with celiac disease. $^{12}$

3. Population-based studies: they are conducted in general population. This study is performed under this modality. This research revealed a prevalence of the disease much higher than expected. Serologic studies in blood donors in Sweden in 1991 showed a prevalence of $1: 256 \cdot{ }^{13}$ Other similar studies indicated the following results: 1:250 in the USA, ${ }^{14} 1: 330$ in the Netherlands, ${ }^{15} 1: 99$ in Finland, ${ }^{16}$ and 1:157 in Israel. ${ }^{17}$ In Mexico a high prevalence of tTG positivity was found in blood donors (1:37). ${ }^{18}$ In Brazil, a study in Sao Pablo showed a prevalence of 1:273, and another in Brasilia of $1: 681 .^{19,20}$

Under the described modality, a study was published in 1993 in Argentina showing a prevalence of 1:1377 in children. The study was conducted using the number of cases diagnosed in hospital centers of the City of Buenos Aires and its suburbs, and the City of La Plata, on the population estimated for that area. ${ }^{21}$ In 1999, a population-based study was conducted on premarital serum samples in the area of La Plata, and a greater prevalence (1:167) was found. ${ }^{22}$

Pediatric studies are scarce. In Finland (2003), a prevalence of 1:99 was found in schoolchildren, ${ }^{23}$ and in Hungary (1999), this was of 1:85. ${ }^{24}$

In Argentina, up to date, no population-based studies have been published on the prevalence in pediatric population.

According to the 2001 Census, Argentina has a predominantly urban distribution of the population $(89 \%){ }^{25}$

\section{OBJECTIVE}

To estimate the prevalence of celiac disease in a pediatric population from 5 urban areas of the Central and Northern regions of Argentina, and to analyze their clinical manifestations, distribution per sex, age group and geographic distribution.

\section{METHODS}

A cross-sectional descriptive design was used. The population was made of children aged 3-16 years old, who attended health-care facilities of five urban districts of the country to have presurgical tests for scheduled or emergency surgeries or fitness tests for sports. We worked with a consecutive sample. Children coming from Gastroenterology, Endocrinology, Rheumatology or that could suffer a pathology related to celiac disease were expressly excluded.

The optimal situation would have been to include children from a general population, but it would have been more difficult to justify the collection of blood among study participants. For this reason, we decided to include such population, given that none of the surgical indications would have relation to celiac disease, and also considering an appropriate and possible population for the objective of the research study. The children had to be Argentineans, with at least one Argentinean parent, so as to minimize the influence of native populations from other countries. We also included those with a previous and accurate diagnosis of celiac disease within that population.

The informed consent of at least one of the parents was obtained.

Seven sites participated: Hospital Materno Infantil de San Isidro, Clínica San Lucas de San Isidro and Hospital Nacional Prof. Dr. A. Posadas (Greater Buenos Aires), CEMIC of the City of 
Buenos Aires, Hospital Infantil of Córdoba, Hospital de Niños de Salta and Hospital de Niños de Santa Fe.

Assuming an expected prevalence of $0.14 \%$ with a $99 \%$ confidence, a sample of 2500 patients was estimated to obtain a semi-amplitude of the confidence interval (CI) of 0.027 .

IgA-anti-tTG antibodies were determined by ELISA (Biosystems Barcelona, Spain) on polystyrene plates coated with calcium-activated recombinant human transglutaminase. Serum dilutions were placed on the plate and confronted after incubation with human/peroxidase anti-IgA conjugate. Dilutions were placed on the ELISA plate reader at $450 \mathrm{~nm}$, with a cutoff of 10 units, in order to quantify reactions.

Positive samples were also tested for IgA EMA by immunofluorescence (IF) with monkey esophagus substrate (Biosystems Barcelona, Spain) and, if positive, a small intestine endoscopic biopsy was proposed after obtaining the informed consent.

Performance of serologic studies was centralized at the Gastroenterology Research Laboratory located in Buenos Aires.

Marsh histopathological criteria were applied, and enteropathies grade II and III were considered compatible with celiac disease. ${ }^{26}$

Prevalence was expressed as a percentage and, when appropriate, with its corresponding exact 95\% CI. Continuous variables were expressed using the mean. Comparison among groups was performed by Fisher's exact test. Results with $p$ values $\leq 0.05$ were considered statistically significant.

The study was approved by the Ethics Committee of the Hospital Materno Infantil de San Isidro.

\section{RESULTS}

Between May 15, 2008 and August 15, 2009 screening of celiac disease by serology was offered to 2230 children. One patient rejected participation and 10 were eliminated due to technical failures in the sample collection. Finally, the population included was of 2219 children (Figure 1), with the following distribution: 480 from Hospital Posadas, 430 from Hospital de Niños de Santa Fe, 397 from Hospital Materno Infantil de San Isidro, 357 from Hospital Municipal Infantil de Córdoba, 308 from Hospital de Niños de Salta, 132 from Sanatorio San Lucas, and 115 from CEMIC.

Children were classified by place of residence in the following groups: Greater Buenos Aires 46\%; Santa Fe 19\%; Córdoba 16\%; Salta 14\%, and City of Buenos Aires 5\%.
The average age (mean) of the population studied was of 8 years and 5 months, with a mean of 8 years.

For analysis, the population was subdivided into three age groups (3-6 y; 7-12 y; 13-16 y).

There were 843 girls (38\%) and 1376 boys $(62 \%)$, since surgeries are more frequent among male children.

As to the causes for blood collection, $89 \%$ were intended for pre-surgical tests, while the rest $(11 \%)$ were for fitness tests.

In total, $96 \%$ of the fathers and $97 \%$ of the mothers were Argentinean. The rest were from bordering countries, such as Uruguay, Bolivia, Paraguay, Chile or Peru, and, to a lesser extent, from USA, Netherlands, Ecuador and Venezuela.

A total of 2219 samples were analyzed for IgAanti-tTG, and IgA EMA was performed on those that turn out positive, with 29 positive samples. One patient had low titer close to the cutoff. Both serologies were consistent in all cases.

We recorded 7 patients from Salta and Córdoba who presented previous diagnosis of celiac disease. These cases were excluded from the serologic analysis, but included as celiac patients in the statistical analysis.

Of the 29 children with positive serologic determinations, 22 small intestine biopsies were performed. The biopsy was not performed on the 7 remaining patients due to family refusal, nor to the patient with low titer for both antibodies. The results of the biopsies performed indicated 1 was normal and 21 were pathological, compatible with celiac disease. The great proportion of severe villus atrophy (n: 20) should be noted, comprising the patients classified as Marsh III. The patient with a normal biopsy was considered as latent celiac disease because of high titers of anti-tTg and positive EMA.

The gluten-free diet was indicated to the 21 patients with celiac disease. Clinical control and later serologic tests were recommended to the latent patient and the boy with low antibody titers.

Among the 843 girls studied, 13 cases were found (1.54\%, CI: $0.82-2.62)$, while 8 cases were found among the 1376 boys (0.82\%, CI: $0.52-1.2)$, $\mathrm{p}=0.039$.

The (mean) age cases diagnosed by histopathology was 9 years and 9 months. Positive histopathology cases were 2/732 patients aged 3-6 y; 12/978 aged 7-12 y and 7/509 aged 13-16 y.

Of the studied population, $4 \%(86 / 2219)$ had a family history of up to second degree of celiac disease and only one new patient $(1 / 21)$ had a 
family history of celiac disease.

Regarding the clinical manifestations of the 21 patients diagnosed by histopathology, the evidence showed a prevalence of the silent form $(66.66 \%)$, followed by the classical symptomatic form $(19 \%)$, and the atypical form $(14 \%)$.

The number of cases of celiac disease found through serologic screening and confirmed by biopsy was 21, and added to the 7 previously diagnosed, the resulting prevalence was of $1.26 \%$ (95\% CI 0.84-1.81) in the studied population. According to these data, the prevalence in this group is of 1 case every 79 children. If we add the patients with positive values and high IgA-anti-tTG and IgA EMA values, who refused to have the biopsy, the prevalence would be of $1.62 \%$ (95\% CI 1.13-2.23), that is to say, 1 case every 61 children (Figure 1).

The analysis of the prevalence by urban districts considering only new cases confirmed by histopathology, showed the following distribution: Hospital Infantil de Córdoba: 1.39\% (95\% CI 0.04-3.22); Hospital de Niños of Salta: 1.29\% (95\% CI 0.35-3.29); sites in the Greater Buenos Aires: 0.99\% (95\% CI 0.47-1.81); and Hospital de Niños de Santa Fe: $0.46 \%$ (95\% CI 0.05-1.66). No cases were found in the City of Buenos Aires.
Table 1 shows the total number of cases, composed by those previously diagnosed and those presenting positive serology, and their distribution by districts. The greatest prevalence was found in the Hospital de Niños de Salta. It is worth mentioning that for 34 out of the 36 cases found were taken into account to determine prevalence. This was because the patient with low antibody titers and the one considered latent were excluded from this analysis.

\section{DISCUSSION}

In 2001, a similar study was published in adult population of La Plata, which estimated a prevalence of $0.6 \%{ }^{22}$ In our study, the prevalence found in this pediatric population reached $1.26 \%$. This increase is remarkable, especially at such age, in which the prevalence is usually lower ${ }^{26}$ The reasons for such increase could be attributed to the fact that the serologic methods used in both studies were different, as well as to the sample design. The IgA-tTG antibody used in this study has greater sensitivity and specificity and is currently the one most used for its simplicity and cost for large studies.

This increasing tendency in prevalence is consistent with the literature. In Finland, Lohi et al.

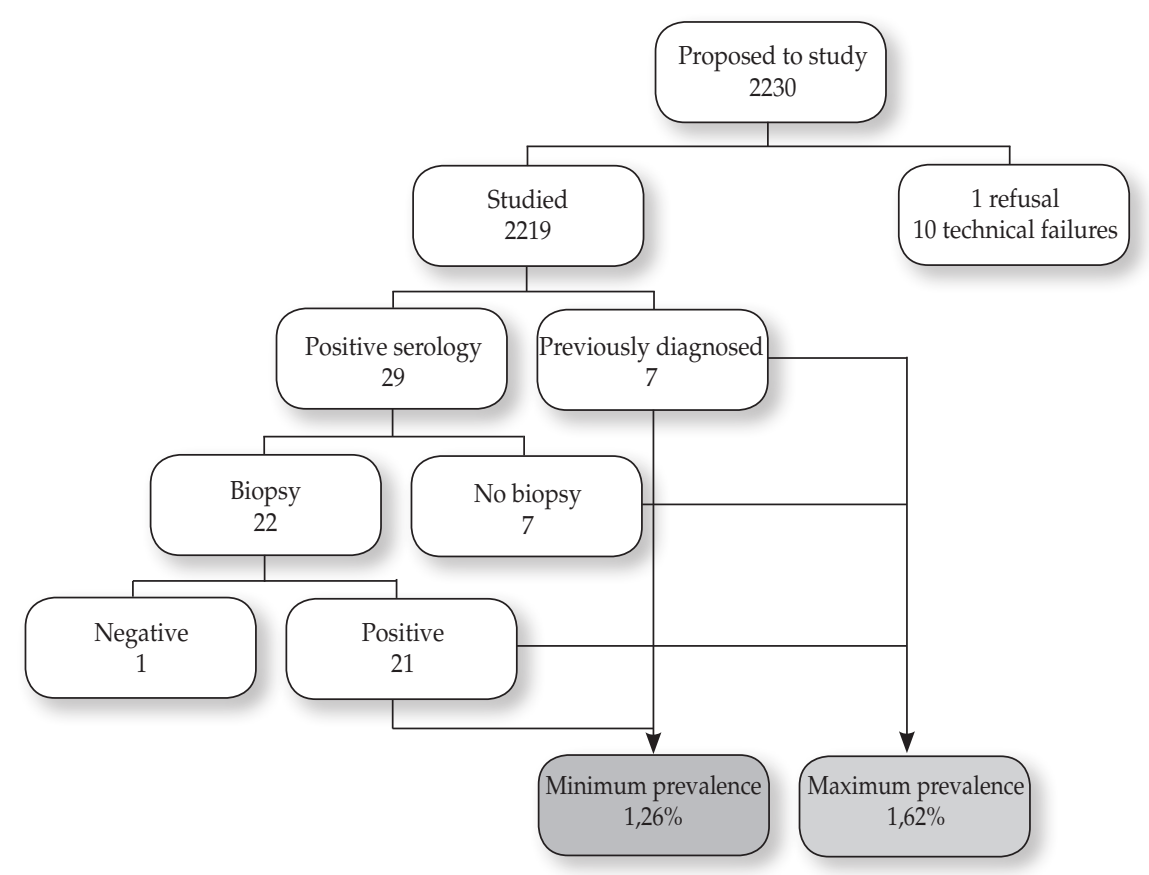


compared 2 population samples separated by a 20-year term, using the same methodology, and observed that, in that period, the prevalence had almost duplicated, which suggests the involvement of other factors, probably environmental, consistent with the increase of other autoimmune diseases. ${ }^{27}$

No total serum IgA and no IgG class antibodies were determined due to their higher costs and low performance. ${ }^{28}$

Consistent with the literature, there was a significant predominance of cases among female patients. ${ }^{29,30}$

A total of $90 \%$ of the positive cases were recorded in the age group corresponding to children of 7 to 16 years old. A growing age-related prevalence was also observed in a study conducted in Spain in pediatric population. ${ }^{31}$

Silent clinical manifestations predominated, though a $33 \%$ of symptomatic cases was detected and almost $20 \%$ were classical forms with nutritional deficiency, chronic diarrhea or abdominal distension, suggesting this is still an under-diagnosed pathology.

Consistent with some publications, ${ }^{32}$ regional differences were found in the global prevalence, including positive serologies and previously diagnosed cases, being greater in the facilities of Salta and Cordoba, and less in Santa Fe.

It should be noted that there was only 1 refusal among the total participations proposed.

Celiac disease meets the criteria proposed by the World Health Organization for screening the general population, because its treatment is effective and there are high sensitivity and specificity tests for asymptomatic cases, but there is no consensus for their recommendation. ${ }^{33}$ Additionally, there is no evidence on the reduction of morbidity and mortality in screened asymptomatic individuals, who also showed less compliance with diet. ${ }^{34}$

Currently, the best guidance in the detection of celiac disease would be to increase the alert in those patients with mild symptoms and in the population at risk. ${ }^{35}$

The presence in this study of a significant number of symptomatic cases suggests the need to increase efforts in the primary health care strategy for a timely detection and an increased knowledge on the whole clinical spectrum of the disease targeted to atypical cases and risk groups, so as to make early diagnoses through the use of serologic determinations, and thus avoiding the long term consequences of the celiac disease.

Strategies to achieve timely detection need to include dissemination of protocols together with scientific information related to the identification of risk groups for professionals from the primary health care area. The asymmetry of the regional prevalence found should be a cause for future studies in more numerous populations in order to confirm such trend.

\section{CONCLUSIONS}

This is the first population-based study on the prevalenceanal of celiac disease in pediatric patients in Argentina. We found a prevalence of $1.26 \%$ (1/79 children) diagnosed by histopathology, which is higher than expected but consistent with other published studies. There was a higher prevalence among girls and $90 \%$ of the cases found were children in over 7 years old.

There were regional differences that should be confirmed by future studies.

The finding of a $33 \%$ of symptomatic cases indicated the need to promote early detection in primary health care.

TABLE 1. Prevalence of children with positive serologies and previously diagnosed cases

\begin{tabular}{lccc}
\hline Urban districts & $\begin{array}{c}\text { Total number of determinations positive } \\
\text { serologies and previously diagnosed cases }\end{array}$ & Prevalence & 95\% IC \\
\hline Hospital Materno Infantil de San Isidro, & $12 / 1009$ & $1.18 \%$ & $0.61-2.06$ \\
Clínica San Lucas, Hopital Posadas & $9 / 358$ & $2.51 \%$ & $1.15-4.71$ \\
Hospital Infantil de Córdoba & $11 / 308$ & $3.57 \%$ & $1.79-6.30$ \\
Hospital de Niños de Salta & $2 / 430$ & $0.46 \%$ & $0.05-1.66$ \\
\hline Hospital de Niños de Santa Fe & & & \\
\hline
\end{tabular}

CI: confidence interval. 


\section{BIBLIOGRAPHY}

1. Dickey W, Kenny BD, McMillan SA, Porter KG, Mc Connel JB, et al. Gastric as well as duodenal biopsies may be useful in the investigation of iron deficiency anemia. Scand J Gastroenterol 1997;32(5):469-72.

2. Stenson WF, Newberry R, Lorenz R, Baldus C, Civitelli R. Increase prevalence of celiac disease and need of routine screening among patients with osteoporosis. Arch Intern Med 2005;165(4):393-9.

3. Van Rijn JC, Grote FK, Oastdijk W, Wit JM. Short stature and the probability of celiac disease in the absence of gastrointestinal symptoms. Arch Dis Child 2004;89:882-3.

4. RostomA,MurrayJ,KagenoffM.AmericanGastroenterological Association (AGA), Institute Technical Review on the diagnosis and management of celiac disease. Gastroenterology 2006;131(6):981-2002.

5. Ventura A, Magazzu G, Greco L. Duration of exposure to gluten and risk of autoimmune disorders in patients with celiac disease. Gastroenterology 1999;117:297-303.

6. Cottone M, Termini A, Olivia L, Magliocco A, et al. Mortality and causes of death in celiac disease in a Mediterranean area. Dig Dis Sci 1999;44:25-38.

7. Bonamico M, Mariani P, Damesi HM, Crisogianni M, Failla $\mathrm{P}$, et al. Prevalence and clinical picture of celiac disease in Italian Down syndrome patients: a multicenter study. J Pediatr Gastroenterol Nutr 2001;33:139-43.

8. Wasertreger S, Litwin N, Mora M. Enfermedad cel'aca asociada a diabetes mellitus insulinodependiente. Arch Argent Pediatr 1996;94(1):10-13.

9. Catassi C, Ratsch IM, Fabianni E, Rossini M. Celiac Disease in the year 2000: exploring de iceberg. Lancet 1994; 343:200-3.

10. Rostom A, DubŽ C, Cranney A, Saloojee N, Sy R, et al. The Diagnostic Accuracy of serologic test for Celiac Disease: a Systematic Review. Gastroenterology 2005;128:S38-46.

11. Davidson LSP, Fountain JR. Incidence of sprue syndrome with some observation on natural history. BMJ 1950; 1: 1157-61.

12. Fasano A, Berti I, Genarduzzi T, Not T, et al. Prevalence of Celiac Disease in at-risk and not risk groups in the United States: a large multicenter study. Arch Intern Med 2003;163(3):286-92.

13. Grodzinsky E, Franzen I, Hed J, Stršm M. High prevalence of celiac disease in healthy adults revealed by antigliadin antibodies. Ann Allergy 1992;69:66-9.

14. Not T, Horvath K, Hill ID, Partanen A, et al. Celiac disease risk in the USA: high prevalence of antiendomysium antibodies in healthy blood donors. Scand J Gastroenterol 1998;33:494-8.

15. Rostami K, Mulder CJ, Werre JM, van Brukelen FR, et al. High prevalence of celiac disease in apparently healthy blood donors suggest a high prevalence of undiagnosed celiac disease in Dutch population. Scand J Gastroenterol 1999;34(3):276-9.

16. Kolho KL, Farkkila MA, Savilahti E. Undiagnosed celiac disease is common in Finnish adults. Scand J Gastroenterol 1998;33:1280-3.

17. Shamir R, Lerner A, Shimar E, Lahat N, et al. The use of a single serological marker underestimates the prevalence of celiac disease in Israel: a study of blood donors. Am J Gastroenterol 2002;97:2589-94.
18. Remes-Troche JM, Ram'rez-Iglesias MT, Rubio-Tapia A, Alonso-Ramos A, et al. Celiac disease could be a frequent disease in Mexico: prevalence of tissue transglutaminase antibody in healthy blood donors. J Clin Gastroenterol 2006;40:697-700.

19. Castanheira SB, Machado MI, Peres LC, Almeida LE, Carvalho L. Prevalence and demographic caracteristics of celiac disease among blood donors in Riberao Preto, State of Sao Paulo, Brazil. Dig Dis and Sci 2006;51(5):1020-5.

20. Gandolfi L, Pratesi R, Cordoba JC, Tauil PL, Gasparin M, et al. Prevalence of celiac disease among blood donors in Brasil. Am J Gastroenterol 2000;95(3):689-92.

21. De RosaS. Estudio multicéntrico para determinar la prevalencia de la enfermedad celíaca en un área de la República Argentina. Med Infant 1993;1(2):72-5.

22. Gómez JC, Selvaggio GS, Viola M, Pizarro B, et al. Prevalence of celiac disease in Argentina: screening of an adult population in La Plata area. Am J Gastroenterol 2001;96 (9):2700-4.

23. Mâki M, Mustalahti K, Kokkonen J, et al. Prevalence of celiac disease among children in Finland. N Eng J Med 2003;348:2517-24.

24. Korponay-Szabó IR, Kovács JB, Czinner A, Gorácz G, et al. High prevalence of silent celiac disease in preschool children screened with IgA IgG antiendomysium antibodies. J Pediatr Gastroenterol Nutr 1999;28(1):26-30.

25. Instituto Nacional de Estadística y Censos (INDEC). Censo Nacional de Población, Hogares y Vivienda 2001.

26. Marsh MN. Gluten, mayor histocompatibility complex and the small intestine: a molecular and immunobiologic approach to the spectrum of gluten sensitivity ("celiac sprue"). Gastroenterology 1992;102:330-54.

27. Lohi S, Mustalahti K, Kaukinen K, Laurila K, et al. Increasing prevalence of celiac disease over time. Aliment Pharmacol Ther 2007;26:1217-25.

28. Lock R, Unsworth D. Identifying Immunoglobulin A deficient children and adults does not necessarily help the serologic diagnosis of celiac disease. J Pediatr Gastroenterol Nutr 1999;28:81-3.

29. Megiorni F, Mora B, Bonamico M, Barbato M, et al. HLADQ and suceptibility to celiac disease: evidence for gender differences and parent of origin effects. Am J Gastroenterol 2008;103:997-1003.

30. Armstrong M, Robins G, Howdle P. Recent advances in celiac disease. Curr Opin Gastroenterol 2009;25:100-9.

31. Castaño L, Blarduni E, Ortiz L, Núñez J, et al. Prospective Screening for Celiac Disease: high prevalence in the first 3 years of life. J Pediatr Gastroenterol Nutr 2004;39:80-84.

32. Olsson C, Stenlund H, Hömell A, Hemell O, Ivarsson A, et al. Regional variation in celiac disease risk within Sweden revealed by the nation wide prospective incidence Register. Acta Paediatr 2009;98(2):337-42.

33. Cataldo F, Accomando S. Celiac disease: to screen or not to screen the general population. J Pediatr Gastroenterol Nutr 2005;40:421-2.

34. Kumar PJ. European and North American populations should be screen for celiac disease. Gut 2003;52:170-1.

35. Berti I, Della Vedova R, Paduano R, Devetta M, et al. Celiac disease in primary care: evaluation of a case-finding strategy. Dis Liver 2006;38(7):461-7. 